\title{
Indentation of polydimethylsiloxane submerged in organic solvents
}

\section{Citation}

Hu, Yuhang, Xin Chen, George M. Whitesides, Joost J. Vlassak, and Zhigang Suo. 2011. "Indentation of Polydimethylsiloxane Submerged in Organic Solvents." Journal of Materials Research 26, no. 6: 785-795.

\section{Published Version}

doi:10.1557/jmr.2010.35

\section{Permanent link}

http://nrs.harvard.edu/urn-3:HUL.InstRepos:12967813

\section{Terms of Use}

This article was downloaded from Harvard University's DASH repository, and is made available under the terms and conditions applicable to Open Access Policy Articles, as set forth at http:// nrs.harvard.edu/urn-3:HUL.InstRepos:dash.current.terms-of-use\#OAP

\section{Share Your Story}

The Harvard community has made this article openly available.

Please share how this access benefits you. Submit a story.

\section{Accessibility}




\title{
Indentation of polydimethylsiloxane submerged in organic solvents
}

Yuhang $\mathrm{Hu},{ }^{1}$ Xin Chen, ${ }^{2}$ George M. Whitesides, ${ }^{2}$ Joost J. Vlassak, ${ }^{1}$ Zhigang Suo 1,a

School of Engineering and Applied Sciences, Kavli Institute, Harvard University, Cambridge, MA 02138

Department of Chemistry and Chemical Biology, Kavli Institute, Harvard University, Cambridge, MA 02138

\begin{abstract}
This paper uses a method based on indentation to characterize a polydimethylsiloxane (PDMS) elastomer submerged in an organic solvent (decane, heptane, pentane, or cyclohexane). An indenter is pressed into a disk of a swollen elastomer to a fixed depth, and the force on the indenter is recorded as a function of time. By examining how the relaxation time scales with the radius of contact, one can differentiate the poroelastic behavior from the viscoelastic behavior. By matching the relaxation curve measured experimentally to that derived from the theory of poroelasticity, one can identify elastic constants and permeability. The measured elastic constants are interpreted within the Flory-Huggins theory. The measured permeabilities indicate that the solvents migrate in PDMS by diffusion, rather than by convection. This work confirms that indentation is a reliable and convenient method to characterize swollen elastomers.
\end{abstract}

a email address: suo@seas.harvard.edu 


\section{INTRODUCTION}

This paper uses a method based on indentation to characterize an elastomeric gel-a network of covalently crosslinked polymers swollen with a solvent. Gels are used in diverse applications, including drug delivery, ${ }^{1-3}$ tissue engineering, ${ }^{4,5}$ microfluidics, ${ }^{6,7}$ and oilfield management. ${ }^{8}$ In a gel, the network can change conformation and enable large and reversible deformation, while the solvent can migrate through the network and enable mass transport. The deformation and mass transport are coupled-a behavior known as poroelasticity.

Most gels are soft, and some are slippery, so that traditional methods for characterizing materials-e.g., tensile and bending tests-are difficult to perform. Furthermore, the change in the conformation of the network and the migration of the solvent result in time-dependent deformation. ${ }^{9}$ These considerations, along with rapidly developing applications of gels, call for the development of reliable and convenient methods to characterize gels.

Indentation has long been used to characterize elasticity and plasticity of metals, ${ }^{10}$ and has in recent years been used to characterize elasticity, ${ }^{11}$ viscoelasticity ${ }^{12,13}$ and poroelasticity ${ }^{14-20}$ of gels. A challenge has been to relate the response of indentation to the properties of the material. When the deformation of a material is time-dependent, the response of indentation depends on how an indenter is pressed into the material. Examples include pressing the indenter at a constant velocity, or at a constant force, or with oscillating depths, or to a fixed depth. Each of these methods of indentation must be analyzed separately, and each has its own merits and difficulties.

This paper adopts a particular method of indentation illustrated in Fig. 1. After an elastomer is saturated with a solvent, an indenter is suddenly pressed into the gel, and 
is subsequently held at a fixed depth $h$. The force on the indenter is recorded as a function of time, $F(t)$. By examining how the relaxation time scales with the radius of contact $a$, we can differentiate the poroelastic behavior from the viscoelastic behavior. By fitting the relaxation curve measured experimentally to that derived from the theory of poroelasticity, we can identify elastic constants and the permeability of the gel.

This method of indentation has been developed recently, ${ }^{18-20}$ but has so far been applied only to a single material, an alginate hydrogel. ${ }^{20}$ To ascertain the effectiveness of the method further, this paper applies the method to an elastomeric network of polydimethylsiloxane (PDMS) submerged in an organic solvent (e.g., decane, heptane, pentane, or cyclohexane) that swells PDMS. PDMS has been used in applications such as microfluidics, where the elastomer may imbibe a solvent and change configuration. ${ }^{21,22}$ We compare our experimental data with the Flory-Huggins theory, and with the data from other experiments in the literature. This work confirms that indentation is an effective method to differentiate the poroelastic and viscoelastic behavior of gels, and to determine the poroelastic properties of gels.

\section{SYNOPSIS OF THE THEORY OF POROELASTICITY}

In our experiment, a disk of the elastomer is submerged in a solvent (Fig. 1). Prior to indentation, the elastomer imbibes the solvent and swells to a state of equilibrium. The process of swelling typically involves finite deformation. Describing such deformation requires a nonlinear field theory. ${ }^{23,24}$ Relative to this homogenous state of the swollen gel, however, the additional strain caused by indentation is small, so long as the depth of indentation is small compared to the radius of contact. Consequently, we will use the swollen gel in the homogeneous state prior to indentation 
as the reference state, and will analyze the time-dependent deformation due to indentation by using a linear theory of poroelasticity. This linear theory was developed by Terzaghi ${ }^{25}$ and Biot ${ }^{26}$ to study the migration of water in soil, and has been adapted to study the migration of a solvent in an elastomer. ${ }^{16,18,24}$ The following synopsis outlines the theory adapted to gels, and the notation to be used in this paper.

Consider a gel in a homogenous state, subject to no mechanical load, with $C_{\mathrm{o}}$ being the number of solvent molecules per unit volume of the gel, and $\mu_{\mathrm{o}}$ the chemical potential of the solvent in the gel. The homogeneous state is taken to be the reference state. When the gel deforms, the displacement is a time-dependent field, $u_{i}\left(x_{1}, x_{2}, x_{3}, t\right)$, and the strain is

$$
\varepsilon_{i j}=\frac{1}{2}\left(\frac{\partial u_{i}}{\partial x_{j}}+\frac{\partial u_{j}}{\partial x_{i}}\right)
$$

The concentration of the solvent in the gel is also a time-dependent field, $C\left(x_{1}, x_{2}, x_{3}, t\right)$. We adopt the convention that specifies the concentration as the number of solvent molecules in the gel divided by the volume of the dry elastomer. The total number of solvent molecules is conserved, so that

$$
\frac{\partial C}{\partial t}+\frac{\partial J_{k}}{\partial x_{k}}=0
$$

where $J_{k}$ is the flux of the solvent.

The gel is in mechanical equilibrium, so that the stress field $\sigma_{i j}\left(x_{1}, x_{2}, x_{3}, t\right)$ satisfies 


$$
\frac{\partial \sigma_{i j}}{\partial x_{j}}=0 .
$$

The gel is not, however, in diffusive equilibrium, so that the chemical potential of the solvent in the gel is a time-dependent field, $\mu\left(x_{1}, x_{2}, x_{3}, t\right)$. The gradient of the chemical potential drives the flux of the solvent according to Darcy's law:

$$
J_{i}=-\left(\frac{k}{\eta \Omega^{2}}\right) \frac{\partial \mu}{\partial x_{i}},
$$

where $\eta$ is the viscosity, and $\Omega$ is the volume per solvent molecule; both are taken to be the values measured when the solvent is in its bulk liquid state. Eq. (4) defines the permeability $k$ as a phenomenological quantity with a unit of length squared.

Each small element of the gel is assumed to be locally in a state of thermodynamic equilibrium, so that the work done on the element equals the change in the free energy, $\delta W=\sigma_{i j} \delta \varepsilon_{i j}+\left(\mu-\mu_{\mathrm{o}}\right) \delta C$, where $W$ is the free energy of the gel divided by the volume of the dry polymer. The individual polymer chains and solvent molecules are commonly assumed to be incompressible. Consequently, the increase in the volume of the gel is entirely due to the additional solvent molecules absorbed, namely,

$$
\varepsilon_{k k}=\Omega\left(C-C_{0}\right)
$$

Under this assumption, the concentration of the solvent is no longer an independent variable, so that the free energy is a function of the six components of the strain. The free energy is taken to be quadratic in the strain:

$$
W=G\left[\varepsilon_{i j} \varepsilon_{i j}+\frac{v}{1-2 v}\left(\varepsilon_{k k}\right)^{2}\right],
$$


where $G$ is the shear modulus and $v$ Poisson's ratio. The equation of state is

$$
\sigma_{i j}=2 G\left(\varepsilon_{i j}+\frac{v}{1-2 v} \varepsilon_{k k} \delta_{i j}\right)-\frac{\mu-\mu_{0}}{\Omega} \delta_{i j}
$$

In the absence of strain, a change in the chemical potential of the solvent in the gel from $\mu_{\mathrm{o}}$ to $\mu$ gives rise to a hydrostatic pressure, $\left(\mu-\mu_{0}\right) / \Omega$, known as the pore pressure in the theory of poroelasticity.

The volume per molecule $\Omega$ and the viscosity $\eta$ are well known for any commonly used solvent. The three other parameters, $G, v, k$, characterize the poroelasticity of a gel. A combination of the above equations gives $\partial C / \partial t=D \nabla^{2} C$, with

$$
D=\frac{2(1-v) G k}{(1-2 v) \eta} .
$$

The field of the solvent concentration obeys the familiar diffusion equation. In the theory of poroelasticity, however, this diffusion equation cannot be solved by itself, because the boundary conditions involve the chemical potential and the displacement. Nonetheless, the diffusion equation indicates that over time $t$ a disturbance diffuses over a length $\sqrt{D t}$.

\section{RELAXATION CURVE DERIVED FROM THE THEORY OF POROELALSTICITY}

We now describe the solution to the poroelastic boundary-value problem associated with indentation, Fig. 1. The solution has a remarkable feature: if the depth of the indentation, $h$, is held fixed, while the force on the indenter relaxes, the radius of 
contact, $a$, remains fixed..$^{18-20}$ In the short-time limit, the concentration of the solvent in the gel remains unchanged, so that $\varepsilon_{k k}=0$. According to the equation of state (7), the gel in the short-time limit behaves like an incompressible elastic material with the shear modulus $G$. Consequently, the solution of an indenter pressed into an incompressible elastic material gives the force in the short-time limit, $F(\mathrm{o})$. In the long-time limit, the gel attains a new state of equilibrium, and the solvent in the gel equilibrates with the external solvent, so that $\mu=\mu_{\mathrm{o}}$. According to the equation of state (7), the gel in the long-time limit behaves like an elastic material with shear modulus $G$ and Poisson's ratio $v$. Consequently, the solution of an indenter pressed into a compressible elastic material gives the force in the long-time limit, $F(\infty)$. The two limits are related by ${ }^{26}$

$$
F(0) / F(\infty)=2(1-v)
$$

For the gel to evolve from the short-time limit toward the long-time limit, the solvent in the gel under the indenter must migrate. The relevant length in this diffusiontype problem is the radius of contact, $a$, and the normalized time takes the form $\tau=D t / a^{2}$. The function $F(t)$ obeys ${ }^{20}$

$$
\frac{F(t)-F(\infty)}{F(0)-F(\infty)}=g(\tau)
$$

The dimensionless ratio on the left-hand side of (10) is a measure of how far the gel is away from the new state of equilibrium. The function $g(\tau)$ must be determined by solving the poroelastic boundary-value problem. Our previous work ${ }^{20}$ indicates that, for a given type of indenter, $g$ is a function of the single variable $\tau$, so long as the radius of contact is much smaller than the size of the disk of the gel. 
For a conical indenter pressed into an incompressible elastic material, the force on the indenter is 27

$$
F(\mathrm{o})=4 G a h
$$

where the radius of contact $a$ relates to the depth of indentation $h$ as

$$
a=\frac{2}{\pi} h \tan \theta
$$

with $\theta$ being half the included angle of the conical indenter. The poroelastic boundaryvalue problem has been solved using the finite element method, giving ${ }^{20}$

$$
g(\tau)=0.493 \exp (-0.822 \sqrt{\tau})+0.507 \exp (-1.348 \tau)
$$

For a spherical indenter of radius $R$, the corresponding relations are

$$
\begin{gathered}
F(\mathrm{o})=\frac{16}{3} G a h, \\
a=\sqrt{R h}, \\
g(\tau)=0.491 \exp (-0.908 \sqrt{\tau})+0.509 \exp (-1.679 \tau) .
\end{gathered}
$$

\section{EXPERIMENTAL DETAILS}

We made the PDMS elastomer by mixing PDMS base and curing agents, at a ratio of 10:1 (by weight) (Essex Brownell, Edison, NJ). The mixture was cured using a cylindrical mold of $5.2 \mathrm{~cm}$ diameter and $1.3 \mathrm{~cm}$ thickness, in an oven at $70^{\circ} \mathrm{C}$ for 4 hours. The curing resulted in a disk of PDMS elastomer. 
We used aluminum indenters of two types: a conical indenter with a half included angle of $\theta=70^{\circ}$, and a spherical indenter with a radius of $20 \mathrm{~mm}$. The indenters were pressed by using a custom-built load frame with a force resolution of $0.01 \mathrm{~N}$ and a displacement resolution of $1 \mu \mathrm{m}$. The indenters were programmed to approach the surface of the elastomer at the speed of $2 \mu \mathrm{m} / \mathrm{s}$, until the slope of the recorded forcedisplacement curve started to be positive. The error in detecting the surface was about 5 $\mu \mathrm{m}$. The indenters were then pressed into the elastomer at a speed of $300 \mu \mathrm{m} / \mathrm{s}$. The time used to press the indenter into the elastomers (2-3 seconds) was much shorter than the relaxation time (tens of seconds for dry elastomers, and hours for swollen elastomers), so that the effect of the initial loading stage was minimized.

To characterize the PDMS elastomer itself, we indented a disk of the elastomer as cured, without imbibing any solvent. Fig. 2(a) shows the recorded relaxation curves at three depths of indentation. We then submerged four disks of the cured PDMS in the organic solvents pentane, heptane, decane, and cyclohexane for more than 48 hours, until the disks were fully saturated with solvent. The dimensions of the swollen disks were measured by a caliper. Each disk swelled by the same ratio in three directions. The swelling ratio $\lambda_{\mathrm{o}}$ of a disk was determined by the diameter of the swollen disk divided by that of the dry disk. The disks were then indented while submerged in the solvents (Fig.

3). For each solvent, the experiment was repeated several times to different depths of indentation (Table I). Figs. 4(a), 5(a) and 6(a) show the recorded relaxation curves.

\section{POROELASTICITY VERSUS VISCOELASTICITY}

Dry PDMS elastomers are known to exhibit viscoelastic behavior, ${ }^{28,29}$ due to conformational changes of the polymer chains. ${ }^{30}$ The rate of viscoelasticity is limited by 
molecular processes such as sliding between the polymer chains, and rotation of the joints between the segments. Consequently, the time for viscoelastic relaxation is independent of the macroscopic length scale, namely, the depth of indentation. According to contact mechanics, the viscoelastic relaxation curve scales as ${ }^{31}$

$$
F=a h f_{v}(t)
$$

where the function $f_{v}(t)$ depends on the viscoelastic material and the shape of the indenter, but is independent of the depth of indentation. ${ }^{29}$

As shown in Fig. 2(b), when the force on the indenter pressed into the dry elastomer is normalized by $a h$, the relaxation curves measured at the three indentation depths collapse into a single curve. That is, the relaxation time of the dry elastomer is independent of indentation depth, a behavior consistent with viscoelastic relaxation. The relaxation time shown in Fig. 2(b) is on the order of tens of seconds, and is within the range of viscoelastic relaxation times reported in the literature. ${ }^{28,29}$

By contrast, an elastomer swollen with a solvent can exhibit both the viscoelastic and the poroelastic behavior. The rate of poroelastic relaxation is limited by the migration of the solvent. As discussed before, when an indenter is pressed into a gel, for the gel to attain the new state of equilibrium, the solvent must migrate, so that the relaxation curve scales as

$$
F=a h f_{p}\left(\frac{t}{a^{2}}\right)
$$

where the function $f_{p}\left(t / a^{2}\right)$ depends on the poroelastic material and the shape of the indenter. That is, the time for poroelastic relaxation scales with $a^{2}$. If the radius of 
contact is large, the time for poroelastic relaxation becomes much longer than the time for viscoelastic relaxation.

As mentioned above, the viscoelastic relaxation time for the dry PDMS elastomer is tens of seconds. When the same PDMS elastomer is saturated with a solvent, the polymer chains are stretched. The viscoelastic relaxation time of the chains is then even shorter. By comparison, when the indenter is pressed into the PDMS elastomeric gels to a depth of hundreds of microns, the relaxation time is on the order of hours, Figs. 4(a) and 5(a). Consequently, for these depths of indentation, the poroelasticity is expected to prevail. Indeed, if the force is normalized by $a h$, but the time is not normalized, the relaxation curves at the three depths of indentation do not collapse into a single curve, Figs. 4(b) and 5(b). However, when the force is normalized by ah, and the time is normalized by $a^{2}$, the relaxation curves for the three depths of indentation collapse into a single curve, Figs. 4(c) and 5(c). This scaling behavior is consistent with poroelastic relaxation.

\section{EXTRACTION OF POROELASTIC CONSTANTS}

By matching the relaxation curve recorded experimentally to that derived from the theory of poroelasticity, we can identify the poroelastic constants $G, v$, and $D$. Take the conical indentation of a PDMS elastomer submerged in heptane, for instance. Inserting the instantaneous force shown in Fig. 4(a) into Eq. (11) gives the shear modulus, $G=0.797 \pm 0.012 \mathrm{MPa}$. A comparison of the experimental value of $F(0) / F(\infty)$ and Eq. (9) yields Poisson's ratio, $v=0.346 \pm 0.003$. As shown in Fig. 4(d), the 
theoretical curve, Eq. 13, overlaps with the experimental data when the diffusivity is taken as $D=2.3 \times 10^{-9} \mathrm{~m}^{2} / \mathrm{s}$.

The same procedure applies to spherical indentation. Comparing the experimental data in Fig. 5(a) with the poroelastic solution for a spherical indenter, Equations (14)-(16), we determine the three poroelastic parameters: $G=0.771 \pm 0.013 \mathrm{MPa}, \quad v=0.327 \pm 0.008$ and $D=3.1 \times 10^{-9} \mathrm{~m}^{2} / \mathrm{s}$. The spherical indentation and the conical indentation were performed on disks of PDMS prepared at different times. The elastic constants determined by the two types of indenters agree well. The diffusivities determined by the two types of indenters differ by $35 \%$. We have not determined whether this difference was caused by any systematic measurement error or by difference in the samples prepared at different times.

The conical indenter was also pressed into PDMS disks submerged in pentane, decane, and cyclohexane. The same scaling relation of $F / a h \sim f_{p}\left(t / a^{2}\right)$ was obtained, Fig. 6. The values of $G, v$, and $D$ obtained for each PDMS-solvent system are listed in Table II.

Thus the indentation-based method determines of both the elastic constants and permeability of gels. Traditional methods like tension, compression, and torsion tests only give the elastic constants. ${ }^{32-34}$ A well-known method to obtain the permeability involves measuring the flux of a solvent through an elastomeric membrane. This method requires eliminating all air bubbles. When the liquid is very volatile the method is even more difficult to operate. It is also difficult to control the homogeneity of the boundary conditions applied on the thin elastomeric memberane. ${ }^{35-42}$ Permeabilities are often derived by measuring the kinetics of swelling. ${ }^{43-46}$ To shorten the time of experiment, swelling is usually carried out by using small spheres, thin fibers, or thin membranes. 
Swelling typically involves large deformation, and quantitative interpretation of experiments is challenging.

\section{INTERPRETATION OF THE ELASTIC CONSTANTS USING THE FLORY-HUGGINS THEORY}

Following Flory and Huggins, ${ }^{47-49}$ we assume that the free energy of a polymeric gel, $W$, is due to stretching the network of the polymer chains and mixing of the polymer chains with the solvent molecules:

$$
W=W_{s}+W_{m}
$$

where $W_{s}$ and $W_{m}$ are the contributions from stretching and mixing, respectively. Let $\lambda_{1}, \lambda_{2}$, and $\lambda_{3}$ be the stretches in the three principal directions. The free energy due to the stretching of a polymeric network is taken to be 47

$$
W_{s}=\frac{1}{2} N k_{B} T\left(\lambda_{1}^{2}+\lambda_{2}^{2}+\lambda_{3}^{2}-3-2 \log \lambda_{1} \lambda_{2} \lambda_{3}\right)
$$

where $N$ is the number of polymer chains in the gel divided by the volume of the dry polymers, and $k_{B} T$ is the temperature in the unit of energy. The free energy of mixing is taken to be 48,49

$$
W_{m}=-\frac{k_{B} T}{\Omega}\left[\Omega C \log \left(1+\frac{1}{\Omega C}\right)+\frac{\chi}{1+\Omega C}\right],
$$

where the concentration of the solvent relates to the stretches as $\Omega C=\lambda_{1} \lambda_{2} \lambda_{3}-1$. The first term inside the bracket represents the entropy of mixing, and the second the 
enthalpy of mixing, where $\chi$ is a dimensionless parameter known as the Flory-Huggins interaction parameter.

Let the network of polymers swell in a solvent until it reaches equilibrium. Take the swelling ratio of the gel in this state to be $\lambda_{1}=\lambda_{2}=\lambda_{3}=\lambda_{0}$. Perturb this state by superimposing a state of small strains $\varepsilon_{11}, \varepsilon_{22}, \varepsilon_{33}$, such that the stretches of the gel become

$$
\lambda_{1}=\lambda_{\mathrm{o}}\left(1+\varepsilon_{11}\right), \lambda_{2}=\lambda_{\mathrm{o}}\left(1+\varepsilon_{22}\right), \lambda_{3}=\lambda_{\mathrm{o}}\left(1+\varepsilon_{33}\right) .
$$

If we expand the free-energy function in a power series up to the second order in the strains, we obtain that

$$
\begin{aligned}
W & =\frac{1}{2} N k_{B} T\left[3\left(1-\frac{1}{\lambda_{\mathrm{o}}^{3}}\right)-\frac{2}{\lambda_{\mathrm{o}}^{3}} \log \lambda_{\mathrm{o}}^{3}\right]-\frac{k_{B} T}{\Omega}\left(1-\frac{1}{\lambda_{\mathrm{o}}^{3}}\right) \log \frac{\lambda_{\mathrm{o}}^{3}}{\lambda_{\mathrm{o}}^{3}-1} \\
& +\left[N k_{B} T\left(\frac{1}{\lambda_{\mathrm{o}}}-\frac{1}{\lambda_{\mathrm{o}}^{3}}\right)-\frac{k_{B} T}{\Omega}\left(\log \frac{\lambda_{\mathrm{o}}^{3}}{\lambda_{\mathrm{o}}^{3}-1}-\frac{1}{\lambda_{\mathrm{o}}^{3}}-\frac{\chi}{\lambda_{\mathrm{o}}^{6}}\right)\right]\left(\varepsilon_{k k}\right) \\
& +\left[\frac{1}{2} N k_{B} T\left(\frac{1}{\lambda_{\mathrm{o}}}-\frac{1}{\lambda_{\mathrm{o}}^{3}}\right)+\frac{1}{2} \frac{k_{B} T}{\Omega}\left(\log \frac{\lambda_{\mathrm{o}}^{3}}{\lambda_{\mathrm{o}}^{3}-1}-\frac{1}{\lambda_{\mathrm{o}}^{3}}-\frac{\chi}{\lambda_{\mathrm{o}}^{6}}\right)\right]\left(\varepsilon_{i j} \varepsilon_{i j}\right) \\
& +\left[\frac{1}{2} \frac{k_{B} T}{\Omega}\left(\frac{1}{\lambda_{\mathrm{o}}^{3}-1}-\log \frac{\lambda_{\mathrm{o}}^{3}}{\lambda_{\mathrm{o}}^{3}-1}-\frac{\chi}{\lambda_{\mathrm{o}}^{6}}\right)\right]\left(\varepsilon_{k k}\right)^{2}
\end{aligned}
$$

A comparison of (23) and (6) gives

$$
\begin{gathered}
\frac{N k_{B} T}{\lambda_{\mathrm{o}}}=G \\
\frac{\lambda_{\mathrm{o}}}{N \Omega}\left(\frac{1}{\lambda_{\mathrm{o}}^{3}-1}-\log \frac{\lambda_{\mathrm{o}}^{3}}{\lambda_{\mathrm{o}}^{3}-1}-\frac{\chi}{\lambda_{\mathrm{o}}^{6}}\right)=\frac{2 v}{1-2 v},
\end{gathered}
$$




$$
N \Omega\left(\frac{1}{\lambda_{\mathrm{o}}}-\frac{1}{\lambda_{\mathrm{o}}^{3}}\right)-\left(\log \frac{\lambda_{\mathrm{o}}^{3}}{\lambda_{\mathrm{o}}^{3}-1}-\frac{1}{\lambda_{\mathrm{o}}^{3}}-\frac{\chi}{\lambda_{\mathrm{o}}^{6}}\right)=0 .
$$

Given $G$ and $v$, the set of nonlinear algebraic equations (24)-(26) determine $\lambda_{\mathrm{o}}, \chi$, and $N$. Fig. 7 plots $\lambda_{\mathrm{o}}$ and $\chi$ as functions of $G$ and $v$. Once $\lambda_{\mathrm{o}}$ is known, $N$ is readily calculated from Eq. (24). At room temperature, $k_{B} T=4 \times 10^{-21} \mathrm{~J}$.

Substituting $G$ and $v$ obtained from the indentation experiments into Eqs. (24)(26), we calculate $\lambda_{0}, \chi$, and $N$ for each PDMS-solvent system, as listed in Table III. The experimentally measured swelling ratios $\lambda_{\mathrm{o}}$ closely reproduce the values reported in the literature. ${ }^{22}$ The directly measured values of $\lambda_{\mathrm{o}}$ differ by less than $5 \%$ from the values calculated using the Flory-Huggins' model. Also listed are the values of $\chi$ found in the literature as obtained from swelling experiments.50-54 The difference between these values and the ones calculated in this paper is less than $15 \%$.

Within the Flory-Huggins theory, the shear modulus of the gel in the dry state is $G_{d r y}=N k_{B} T .47 \quad$ For every PDMS-solvent system, the calculated $N k_{B} T$ should be the same and equal the shear modulus of the dry PDMS. Observe that $G_{d r y}$ can be determined from the measured relaxation curve on the cured PDMS elastomer without any solvent, Fig. 2. Assuming incompressibility of the dry PDMS elastomer and substituting the long-time force shown in Fig. 2 into the analytical relation $F(\infty) / a h=4 G_{d r y}$ yields $G_{d r y}=1.01 \pm 0.02 \mathrm{MPa}$. Table III lists the values of $N k_{B} T$ for the four PDMS-solvent systems calculated by using Eq. (24) and the measured values of the shear modulus and swelling ratio. These values, $N k_{B} T=1.07 \pm 0.06 \mathrm{MPa}$, are consistent with the experimental value of $G_{d r y}$. 


\section{CONVECTION VERSUS DIFFUSION}

Darcy's law, Eq. (4), is phenomenological, and is not tied to any specific mechanism of transport. Similarly, the quantity defined by Eq. (8) is named diffusivity purely by its macroscopic effect; this name does not imply that solvent molecules must diffuse in the network. Indeed, the theory of Terzaghi ${ }^{25}$ and Biot ${ }^{26}$ were introduced for the flow of bulk water through soil. A solvent can migrate through a polymer network by convection or diffusion. Convection describes solvent molecules moving collectively as a bulk liquid, and is expected to occur when the mesh size of the network is much larger than the size of an individual solvent molecule. By contrast, diffusion describes solvent molecules moving individually at random, and is expected to occur when the mesh size of the network is comparable to the size of an individual solvent molecule.

Listed in Table II are values of self-diffusivity of the solvents in the bulk liquid state independently measured by other researchers. ${ }^{55-57}$ These values are close to our measured diffusivities for the solvents migrating in the PDMS. The average mesh size $\xi$ of a polymeric network is estimated by 58,59

$$
\frac{4}{3} \pi\left(\frac{\xi}{2}\right)^{3}=\frac{1}{N / \lambda_{0}^{3}} .
$$

Inserting the experimental swelling ratio $\lambda_{0}$ which was directly measured using caliper (Table III) and the shear modulus of the dry PDMS elastomer, $G_{d r y}=1.01 \pm 0.02 \mathrm{MPa}$ into Eqs. (24) and (27), we estimate the mesh size of the swollen PDMS gels in each organic solvent. The calculated values are listed in Table III. The estimated mesh size of the swollen PDMS gels is on the same order of magnitude as the dimension of the solvent. 
Thus, it is not surprising that the solvents migrate in the PDMS elastomer by diffusion. Fig. 8 shows a linear relation between the measured diffusivity $D$ and the inverse of the viscosity $1 / \eta$. This relation has also been observed for organic solvents permeating through membranes of swollen elastomers. 35

For each PDMS-solvent system, the permeability is calculated according to (8) and listed in Table II. The correlation of permeability with the molecular volume of the solvent is plotted in Fig. 9. For solvents of chain-like molecules such as pentane, heptane, and decane, the permeability of PDMS gels decreases as the molecular volume of the solvent increases. The same trend has been obtained for the permeability of alcohols through porous glass. ${ }^{60}$ Also evident in Fig. 9 is that the permeability of cyclohexane does not follow the same trend as for chain-like molecules. That is, the permeability is also affected by the shape of the solvent molecule. This result offers another piece of evidence that the mechanism of solvent transport in the swollen PDMS is diffusive.

Incidentally, we previously used the same method to study the migration of water in a covalently cross-linked alginate hydrogel. ${ }^{20}$ The measured diffusivity was $3.24 \times 10^{-8} \mathrm{~m}^{2} / \mathrm{s}$, which was two orders of magnitude larger than the self-diffusivity of water $8 \times 10^{-10} \mathrm{~m}^{2} / \mathrm{s}$. The mesh size of the alginate gel is $11.6 \pm 0.4 \mathrm{~nm},{ }^{61}$ which is approximately forty times the dimension of the water molecule $2.8 \AA$. Consequently, water is expected to migrate in the alginate hydrogel by convection.

\section{CONCLUDING REMARKS}

While time-dependent and finite deformation of gels is difficult to analyze in general, the additional strain superimposed on swollen gels by indentation is small, and 
can be analyzed by using the linear theory of poroelasticity. The solution to the boundary-value problem takes a remarkably simple form, enabling indentation to be a convenient and reliable method to study the mechanics, thermodynamics and kinetics of gels. The experiments readily differentiate mechanisms of deformation (viscoelasticity and poroelasticity). The experiments also readily differentiate mechanisms of transport (convection and diffusion). By fitting the relaxation curve measured experimentally to that derived from the theory of poroelasticity, we can identify elastic constants and the permeability of. The measured elastic constants of the PDMS-solvent systems can be interpreted by using the Flory-Hugging theory. The measured permeabilities suggest that the organic solvents migrate in the PDMS by diffusion, rather than by convection.

\section{ACKNOWLEDGEMENT}

This work is supported by the NSF (CMMI-o800161), MURI (W911NF-09-10476), and MRSEC at Harvard University. 


\section{References}

1. R. Duncan: The dawning era of polymer therapeutics. Nat. Rev. Drug Discovery 2, 347 (2003).

2. B. Jeong, Y.H. Bae, D.S. Lee, and S.W. Kim: Biodegradable block copolymers as injectable drug-delivery systems. Nature 388, 860 (1997).

3. R. Langer: Drug delivery and targeting. Nature 392, 5 (1998).

4. Y. Luo, and M.S. Shoichet: A photolabile hydrogel for guided three-dimensional cell growth and migration. Nat. Mater. 3, 249 (2004).

5. A.P. Nowak, V. Breedveld, L. Pakstis, B. Ozbas, D.J. Pine, D. Pochan, and T.J. Deming: Rapidly recovering hydrogel scaffolds from self-assembling diblock copolypeptide amphiphiles. Nature 417, 424 (2002).

6. D.J. Beebe, J.S. Moore, J.M. Bauer, Q. Yu, R.H. Liu, C. Devadoss, and B.H. Jo: Functional hydrogel structures for autonomous flow control inside microfluidic channels. Nature 404, 588 (2000).

7. M. Tokeshi, T. Minagawa, K. Uchiyama, A. Hibara, K. Sato, H. Hisamoto, and T. Kitamori: continuous-flow chemical processing on a microchip by combining microunit operations and a multiphase flow network. Ana. Chem. 74, 1565 (2002).

8. S. Cai, Y. Lou, P. Ganguly, A. Robisson, and Z. Suo: Force generated by a swelling elastomer subject to constraint. J. Appl. Phys. 107, 103535 (2010).

9. X. Zhao, N. Huebsch, D.J. Mooney, and Z. Suo: Stress-relaxation behavior in gels with ionic and covalent crosslinks. J. Appl. Phys. 107, 063509 (2010). 
10. W.C. Oliver, and G.M. Pharr: An improved technique for determining bardness and elastic modulus using load and displacement sensing indentation experiments. $J$. Mater. Res. 7, 1564 (1992).

11. D.M. Ebenstein, and L.A. Pruitt: Nanoindentation of soft hydrated materials for application to vascular tissues. J. Biomed. Mater. Res. A 69, 222 (2004).

12. J.D. Kaufman, G.J. Miller, E.F. Morgan and C.M. Klapperich: Time-dependent mechanical characterization of poly(2-hydroxyethyl methacrylate) hydrogels using nanoindentation and unconfined compression. J. Mater. Res. 23, 1472 (2008).

13. G. Constantinides, Z.I. Kalcioglu, M. McFarland, J.F. Smith, and K.J. Van Vliet: Probing mechanical properties of fully hydrated gels and biological tissues. $J$. Biomech. 41, 3285 (2008).

14. M. Galli, K.S.C. Comley, T.A.V. Shean, and M.L. Oyen: Viscoelastic and poroelastic mechanical characterization of hydrated gels. J. Mater. Res. 24, 973 (2009).

15. M. Galli, and M.L. Oyen: Spherical indentation of a finite poroelastic coating. Appl. Phys. Lett. 93, 031911 (2008)

16. M. Galli and M. L. Oyen: Fast indentation of poroelastic parameters from indentation tests. CMES 48, 241 (2009).

17. W.C. Lin, K.R. Shull, C.Y. Hui and Y.Y. Lin: Contact measurement of internal fluid flow within poly(n-isopropylacrylamide) gels. J. Chem. Phys. 127, 094906 (2007).

18. C.Y. Hui, Y.Y. Lin, F.C. Chuang, K.R. Shull, and W.C. Ling: A contact mechanics method for characterizing the elastic properties and permeability of gels. $J$. Polymer Sci B: Polymer Phys. 43, 359 (2006). 
19. Y.Y. Lin, B.W. Hu: Load relaxation of a flat rigid circular indenter on a gel half space. J. Non-Crystalline Solids 352, 4034 (2006).

20. Y. Hu, X. Zhao, J.J. Vlassak, Z. Suo: Using indentation to characterize the poroelasticity of gels. Appl. Phys. Lett. 96, 121904 (2010).

21. M.W. Toepke, and D.J. Beebe: PDMS absorption of small molecules and consequences in microfluidic applications, Lab chip 6, 1484 (2006).

22. J.N. Lee, C. Park, and G.M. Whitesides: Solvent compatibility of poly(dimethylsiloxane)-based microfluidic devices. Anal. Chem. 75, 6544 (2003).

23. W. Hong, X. Zhao, J. Zhou, and Z. Suo: A theory of coupled diffusion and large deformation in polymeric gels. J. Mech. Phys. Solids 56, 1779 (2008).

24. M. Doi: Gel dynamics. J. Phys. Soc. Jpn. 78, 052001 (2009).

25. K. Terzaghi : Die berechnung der durchlässigkeitsziffer des tones aus dem verlauf der hydrodynamischen spannungsercheinungen. Sitzungsber. Akad. Wiss. Wien Math. -Naturewiss. Kl., Abt. IIa 132, 125 (1923).

26. M.A. Biot: General theory of three-dimensional consolidation. J. Appl. Phys. 12, 155 (1941).

27. I.N. Sneddon: The relation between load and penetration in the axisymmetric Boussinesq problem for a punch of arbitrary profile. Int. J. Engng Sci. 3, 47 (1965).

28. M.R. Vanlandingham, N.-K. Chang, P.L. Drzal, C.C. White, and S.-H Chang: Viscoelastic characterization of polymers using instrumented indentation. I. quasistatic testing. J. Polym. Sci. Part B: Polym. Phys. 43, 1794 (2005). 
29. I.-K Lin, K.-S. Ou, Y.-M. Liao, Y. Liu, K.-S. Chen, and X. Zhang: Viscoelastic characterization and modeling of polymer transducers for biological applications. $J$. Microelectromech. Syst. 18, 1087 (2009).

30. J.D. Ferry: Viscoelastic properties of polymers, $3^{\text {rd }}$ ed. (John Wiley and Sons, New York, NY, 1980).

31. K.J. Johnson: Contact mechanics. (Cambridge University Press, New York, NY, 1987).

32. E. Geissler, and A.M. Hecht: The Poisson ratio in polymer gels. 2. Macromolecules 14, 185 (1981).

33. S. Hirotsu: Softening of bulk modulus and negative Poisson's ratio near the volume phase transition of polymer gels. J. Chem. Phys. 94, 3949 (1991).

34. J. Tang, M.A. Tung, J. Lelievre, and Y. Zeng: Stress-strain relationships for gellan gels in tension, compression and torsion. J. Food Eng. 31, 511 (1997).

35. D.R. Paul and O.M. Ebra-Lima: Pressure-induced diffusion of organic liquids through highly swollen polymer membranes. J. Appl. Polym. Sci. 14, 2201 (1970).

36. D.R. Paul and O.M. Ebra-Lima: The mechanism of liquid transport through swollen polymer membranes. J. Appl. Polym. Sci. 15, 2199 (1971).

37. D.R. Paul: Further comments on the relation between hydraulic permeation and diffusion. J. Polym. Sci.: Polym. Phys. Ed. 12, 1221 (1974).

38. D.R. Paul: Reformulation of the solution-diffusion theory of reverse osmosis. $J$. Membr. Sci. 241, 371 (2004). 
39. P. Meares: On the mechanism of desalination by reversed osmotic flow through cellulose acetate membranes. Eur. Polym. J. 2, 241 (1966).

40. A. Peterlin and H. Yasuda, Comments on the relation between hydraulic permeability and diffusion in homogeneous swollen membranes. J. Polym. Sci., Polym. Phys. Ed. 12, 1215 (1974).

41. H. Yasuda, and A. Peterlin: Diffusive and bulk flow transport in polymers. J. Appl. Polym. Sci. 17, 433 (1973).

42. J.G. Wijmans, and R.W. Baker: The solution-diffusion model: a review. J. Membr. Sci. 107, 1 (1995).

43. T. Tanaka, and D.J. Fillmore: Kinetics of swelling of gels. J. Chem. Phys. 70, 1214 (1979).

44. Y. Li, and T. Tanaka: Kinetics of swelling and shrinking of gels. J. Chem. Phys. 92, 1365 (1990).

45. T. Yamaue, and M. Doi: Swelling dynamics of constrained thin-plate gels under an external force. Phys. Rev. E 70, 011401 (2004).

46. T. Hajsz, I. Csetneki, G. Filipcsei, and M. Zrinyi: Swelling kinetics of anisotropic filler loaded PDMS networks. Phys. Chem. Chem. Phys. 8, 977 (2006).

47. P.J. Flory: Principles of polymer chemistry. (Cornell University, Ithaca, 1953).

48. P.J. Flory: Thermodynamics of high polymer solutions. J. Chem. Phys. 10, 51 (1942).

49. M.L. Huggins: Solutions of long chain compounds. J. Chem. Phys. 9, 440 (1941). 
50. M. Braden, D. Latham, and M.P. Patel: Observations on the swelling of cross-linked poly(dimethylsiloxane) networks by solvents. Eur. Polym. J. 41, 3069 (2005).

51. M. Gottlieb, and M. Herskowitz: Estimation of the $\chi$ parameter for poly(dimethylsiloxane) solutions by the UNIFAC group contribution method. Macromolecules 14, 1468 (1981).

52. P.J. Flory: Thermodynamics of polymer solutions. Fifteenth spiers memorial lectures. Discuss. Faraday Soc. 49, 7 (1970).

53. P.J. Flory, and H. Shih: Thermodynamics of solutions of poly(dimethylsiloxane) in Benzene, Cyclohexane, and Chlorobenzene. Macromolecuels 5, 761 (1972).

54. N. Kuwahara, T. Okazawa, and M. Kaneko: Osmotic pressures of moderately concentrated polydimethylsiloxane solutions. J. Polym. Sci. Part C 23, 543 (1968).

55. D.C. Douglass, and D.W. McCall: Diffusion in paraffin hydrocarbons. J. Phys. Chem. 62, $1102(1958)$.

56. E. Fishman: Self-diffusion in liquid n-pentane and n-heptane. J. Phys. Chem. 59, 469 (1955)

57. M. Holz, S.R. Heil, and A. Sacco: Temperature-dependent self-diffusion coefficients of water and six selected molecular liquids for calibration in accurate $\mathrm{H}$ NMR PFG measurements. Phys. Chem. Chem. Phys. 2, 4740 (2000).

58. J. Schurz: Rheology of polymer solutions of the network type. Prog. Polym. Sci. 16, 1 (1991). 
59. M. Grassi, C. Sandolo, D. Perin, T. Coviello, R. Lapasin, and G. Grassi: Structural characterization of calcium alginate matrices by means of mechanical and release tests. Molecules 14, 3003 (2009).

6o. W.V. Vadakan and G.W. Scherer: Measuring permeability of rigid materials by a beam-bending method: II, porous glass. J. Am. Ceram. Soc. 83, 2240 (2000).

61. T. Boontheekul, H.-J. Kong, D.J. Mooney: Controlling alginate gel degradation utilizing partial oxidation and bimodal molecular weight distribution. Biomaterials 26, 2455 (2005). 
TABLE I. Type of indenter and depths pressed into each sample.

\begin{tabular}{ccccc}
\hline \hline samples & Type of & & \\
& indenter & & \\
& & & \\
& cone & 400 & 700 & 1000 \\
Dry PDMS & & & & \\
cone & 600 & 800 & 1000 \\
PDMS gel in decane & cone & 600 & 800 & 1000 \\
& sphere & 200 & 300 & 400 \\
PDMS gel in heptane & & & & \\
& cone & 400 & 600 & 800 \\
PDMS gel in pentane & & & & \\
& & 600 & 800 & 1000 \\
\hline \hline
\end{tabular}


TABLE II. Poroelastic properties of each PDMS/solvent system and the properties of the solvent used in each system.

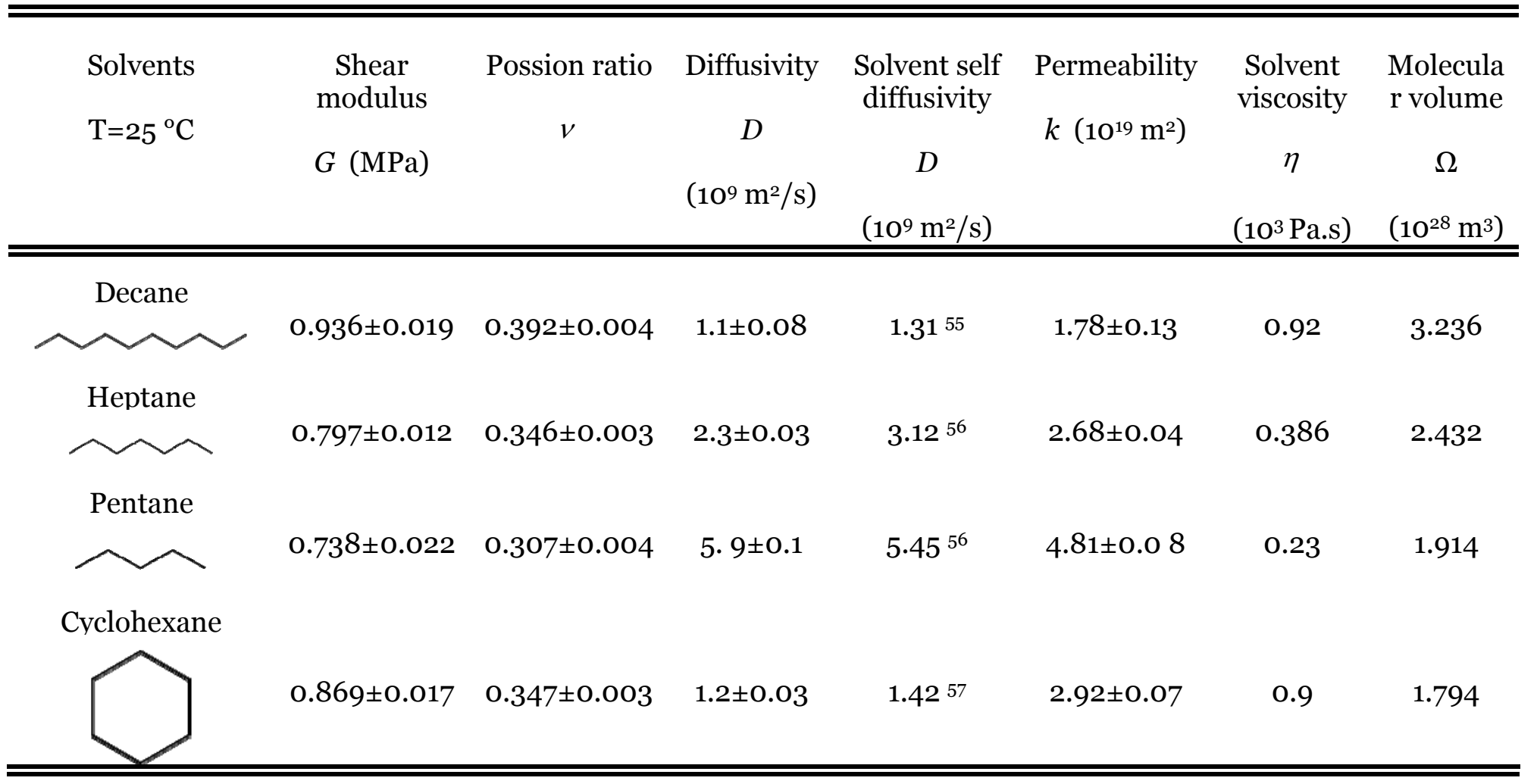


TABLE III. Thermodynamic properties of each PDMS-solvent system

\begin{tabular}{|c|c|c|c|c|c|c|}
\hline $\begin{array}{l}\text { Solvents } \\
\mathrm{T}=25^{\circ} \mathrm{C}\end{array}$ & $\begin{array}{c}\text { Measured } \\
\text { swelling } \\
\text { ratio } \\
\lambda_{\mathrm{o}}\end{array}$ & $\begin{array}{c}\text { Swelling } \\
\text { ratio } \\
\lambda_{\mathrm{o}}\end{array}$ & $\begin{array}{l}N k_{B} T \\
(\mathrm{MPa})\end{array}$ & $\begin{array}{c}\text { Florry- } \\
\text { Huggins } \\
\text { parameter } \\
\chi\end{array}$ & $\begin{array}{l}\text { Literature } \\
\qquad \chi\end{array}$ & $\begin{array}{c}\text { Mesh size, } \\
\xi \\
\left(10^{9} \mathrm{~m}\right)\end{array}$ \\
\hline Decane & 1.26 & 1.19 & 1.11 & 0.8 & & 2.47 \\
\hline Heptane & 1.33 & 1.28 & 1.02 & 0.66 & $0.53^{-0.64^{50-52}}$ & 2.61 \\
\hline Pentane & 1.42 & 1.37 & 1.01 & 0.57 & $0.51-0.59^{50-52}$ & 2.79 \\
\hline Cyclohexane & 1.32 & 1.31 & 1.13 & 0.65 & $0.51-0.55^{53,54}$ & 2.59 \\
\hline
\end{tabular}



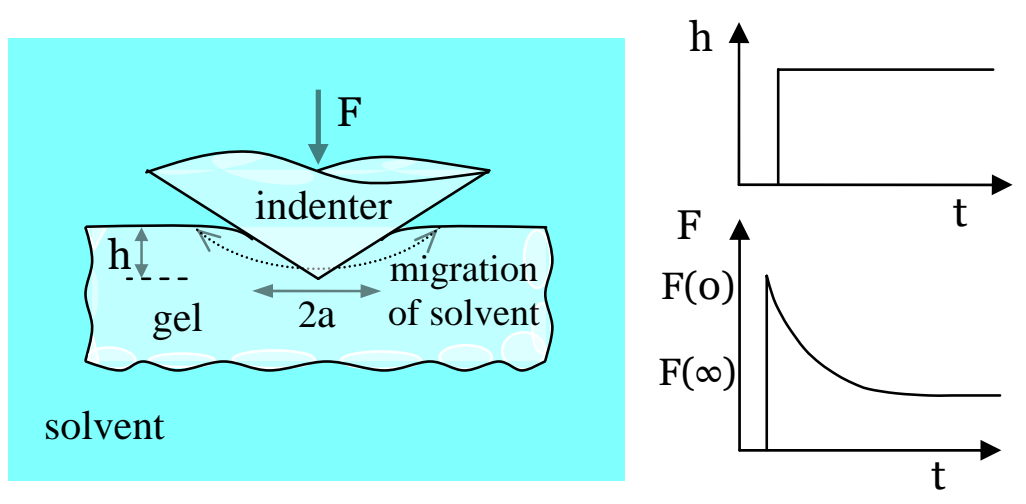

FIG. 1. A disk of a gel is submerged in a solvent throughout the experiment. Prior to indentation, the gel imbibes the solvent and swells to a state of equilibrium. An indenter is then rapidly pressed into the gel, and is subsequently held at a fixed depth $h$. The indenter causes the solvent to migrate in the gel, so that the force $F$ on the indenter decreases as a function of time, until the gel reaches a new state of equilibrium. 

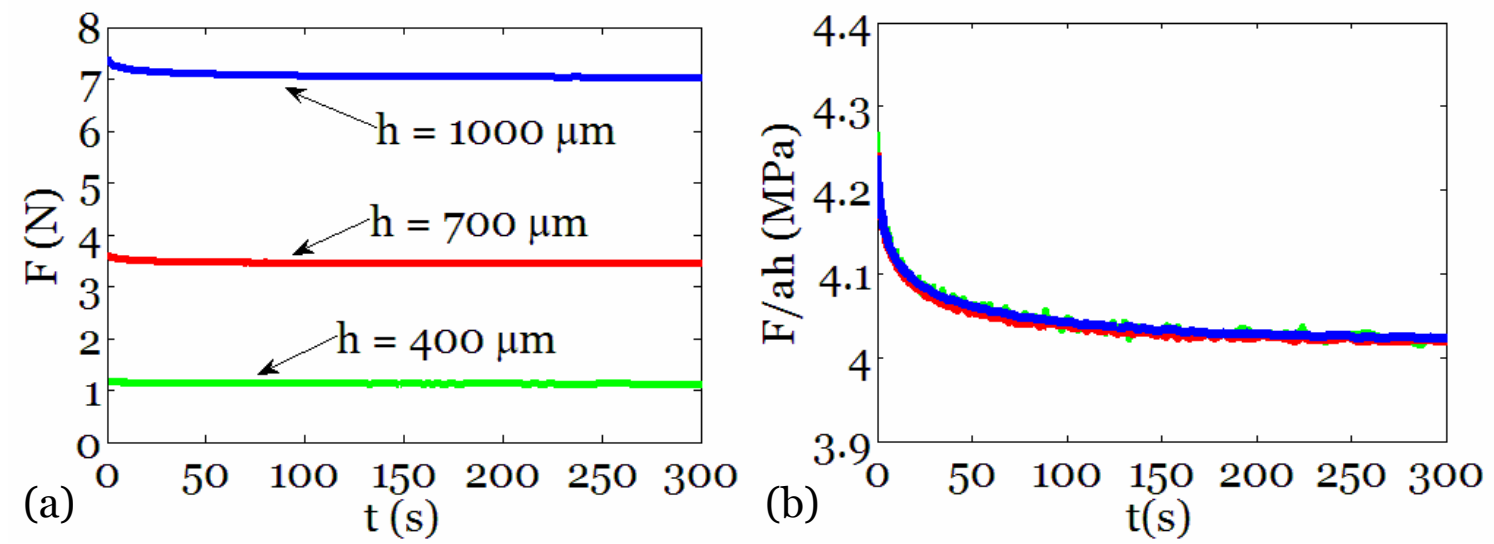

FIG. 2. A conical indenter was pressed into a dry PDMS sample to three depths. (a) Experimentally measured force as a function of time. (b) When the force is normalized as $F / a h$, but the time is not normalized, the force-relaxation curves for the three depths of indentation collapse to a single curve. 

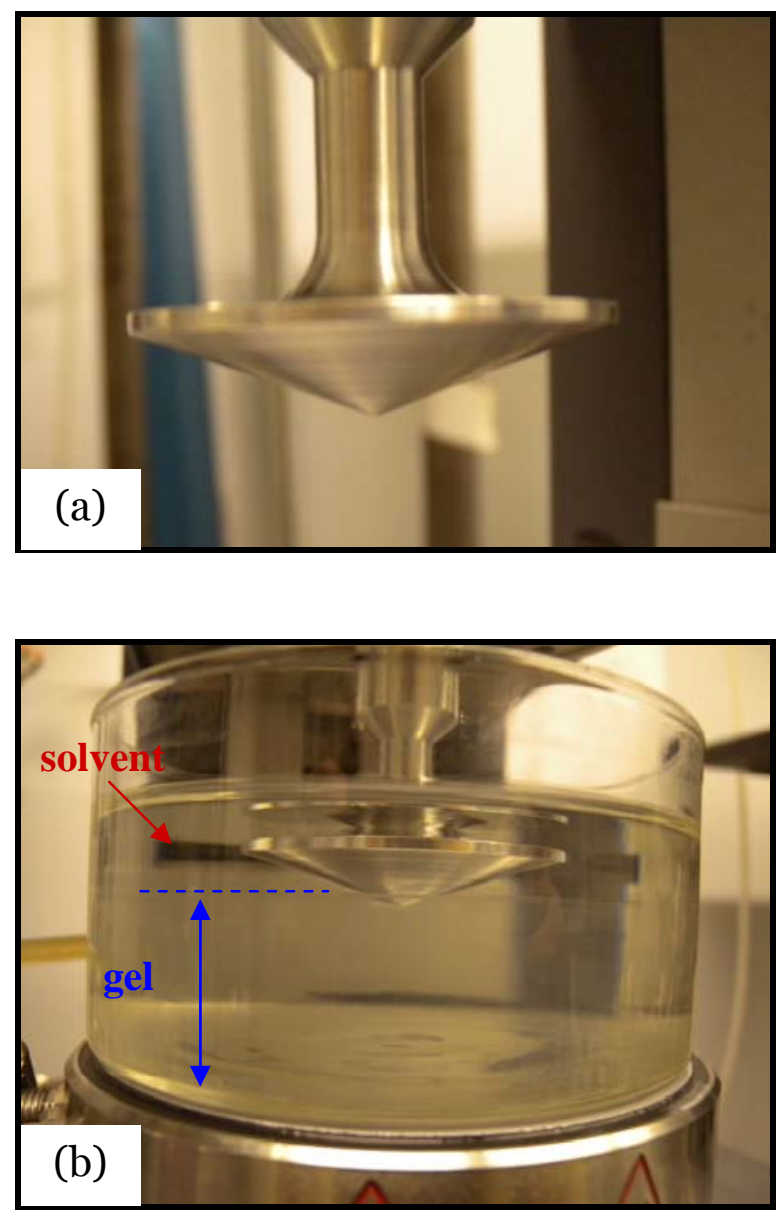

FIG. 3. Experimental setup. (a) A conical indenter made of aluminum. (b) The indenter is pressed into a disk of PDMS, which is submerged in a solvent. 

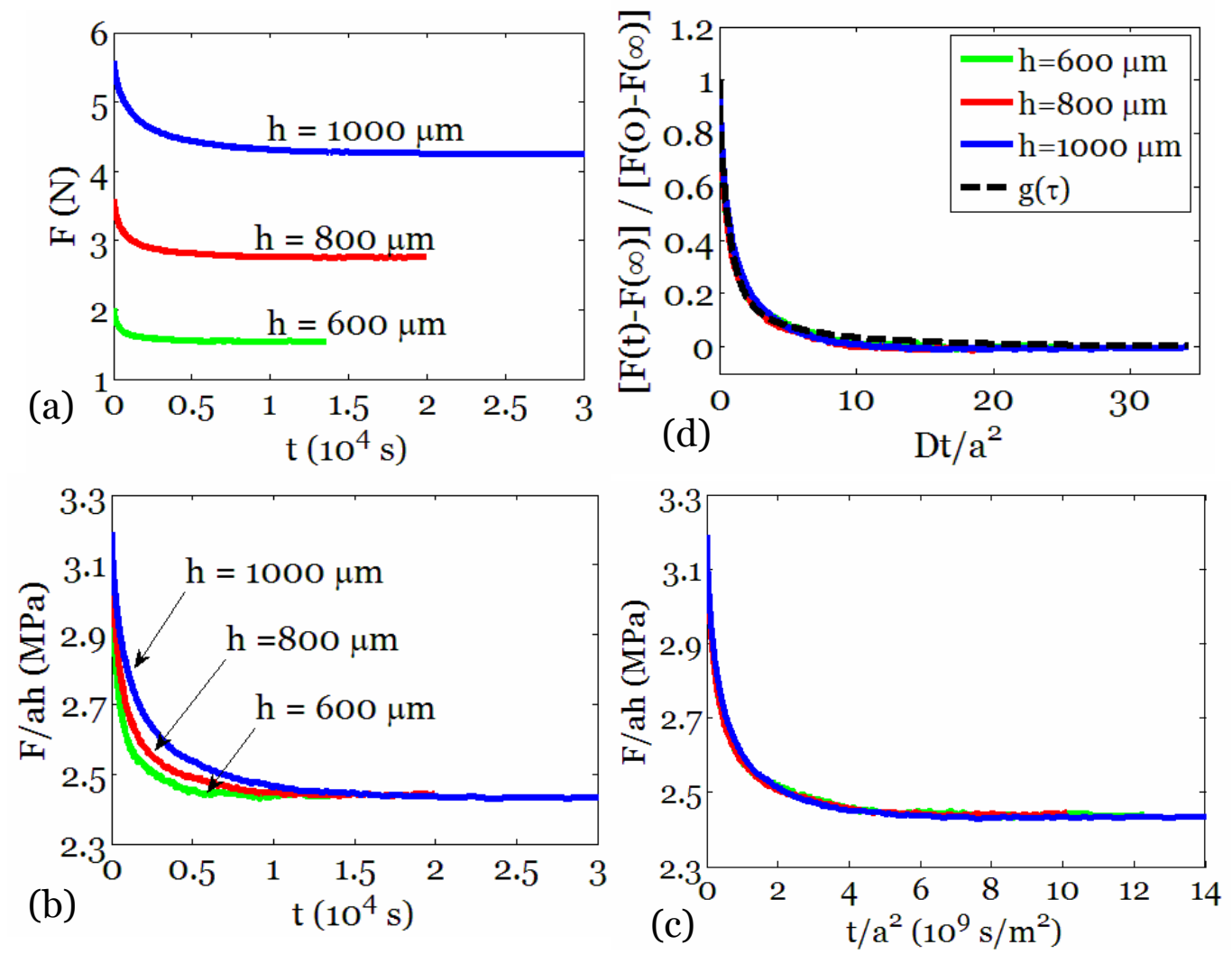

FIG. 4. A conical indenter was pressed to three depths into a PDMS gel submerged in heptane. (a) Experimentally measured force as a function of time. (b) The force is normalized as $F / a h$, but the time is not normalized. (c) The force is normalized as $F / a h$, and the time is normalized as $t / a^{2}$. (d) The force is normalized as $[F(t)-F(\infty)] /[F(0)-F(\infty)]$, and the time is normalized as $D t / a^{2}$. 

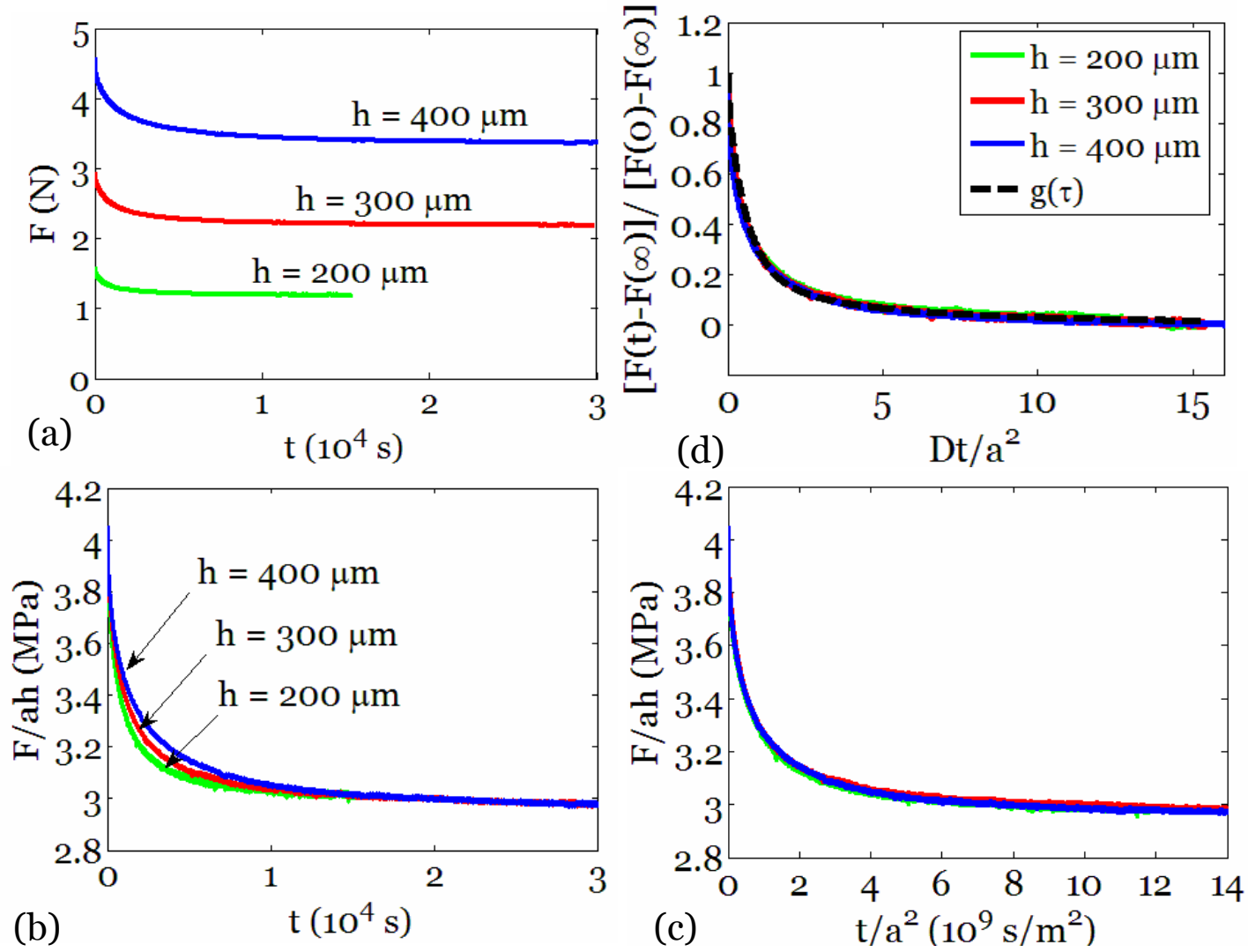

FIG. 5. A spherical indenter was pressed to three depths into a PDMS gel submerged in heptane. (a) Experimentally measured force as a function of time. (b) The force is normalized as $F / a h$, but the time is not normalized. (c) The force is normalized as $F / a h$, and the time is normalized as $t / a^{2}$. (d) The force is normalized as $[F(t)-F(\infty)] /[F(0)-F(\infty)]$, and the time is normalized as $D t / a^{2}$. 

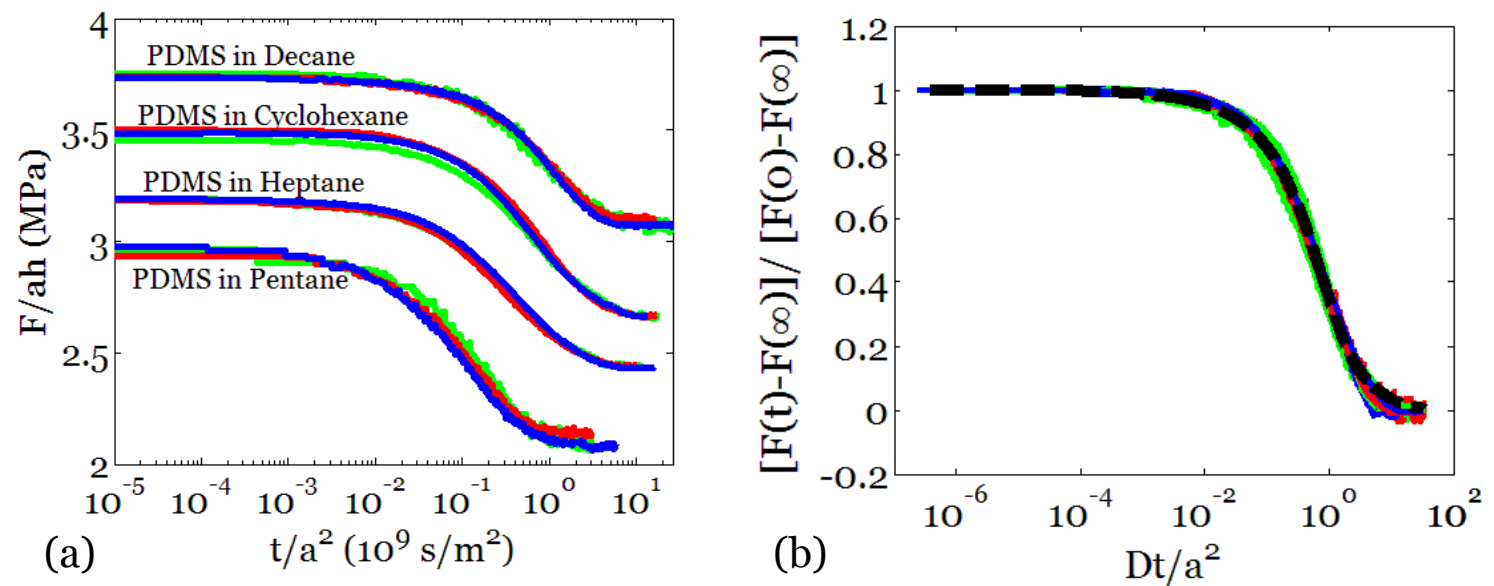

FIG. 6. A conical indenter was pressed to three depths into four disks of PDMS submerged in different solvents. (a) The force is normalized as $F / a h$, and the time is normalized as $t / a^{2}$. (b) The force is normalized as $[F(t)-F(\infty)] /[F(\mathrm{o})-F(\infty)]$, and the time is normalized as $D t / a^{2}$. For the experimental data to fit the function $g(\tau)$, each solvent needs a distinct value of $D$, as listed in Table 2 . 

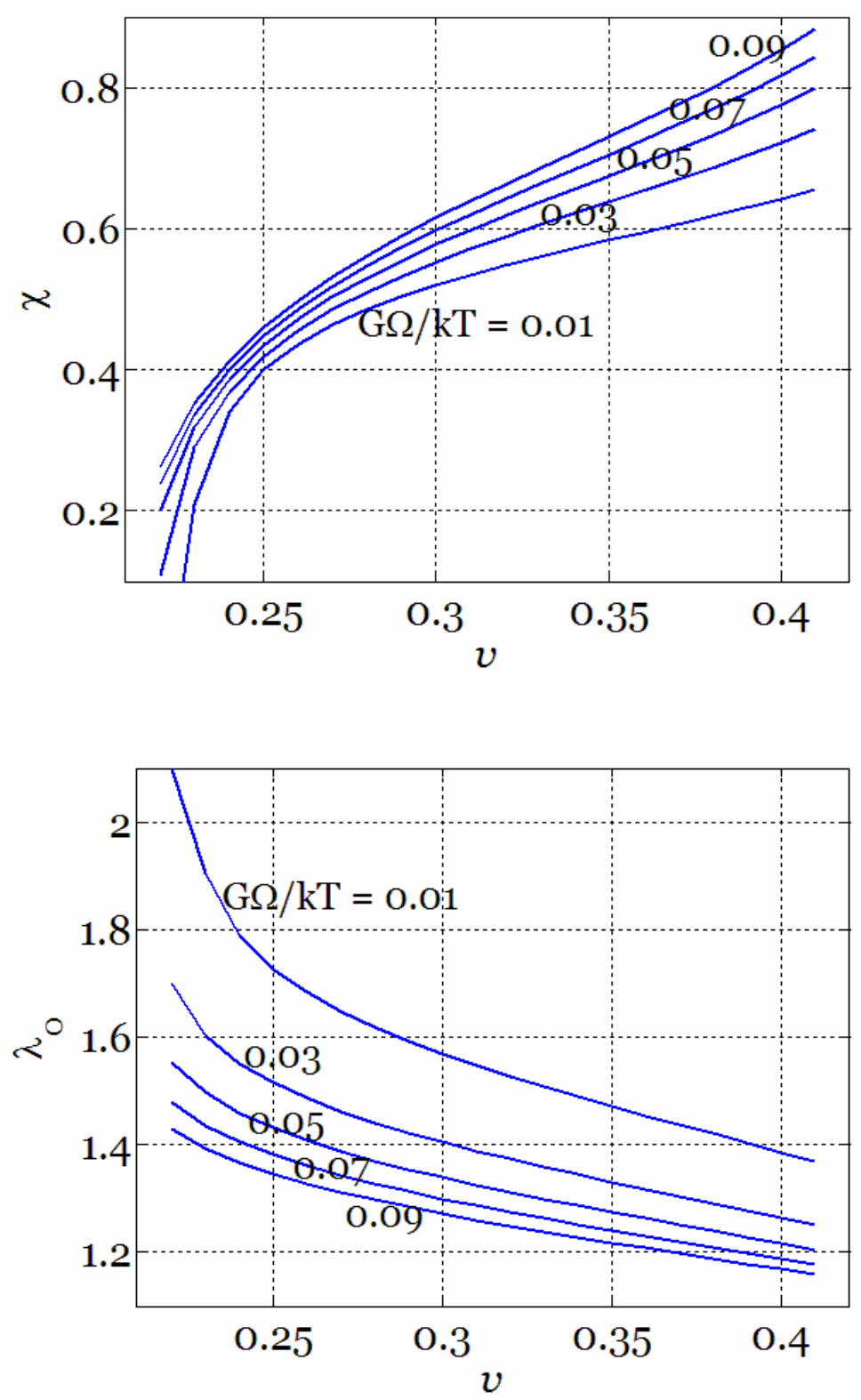

FIG. 7. Thermodynamic parameters are expressed in poroelastic parameters. (a) FloryHuggins interaction parameter $\chi$ vs. Possion's ratio $v$ for various normalized shear modulus $G \Omega / k T$. (b) Swelling ratio $\lambda_{\mathrm{o}}$ vs. Possion's ratio $v$ for various $G \Omega / k T$. 


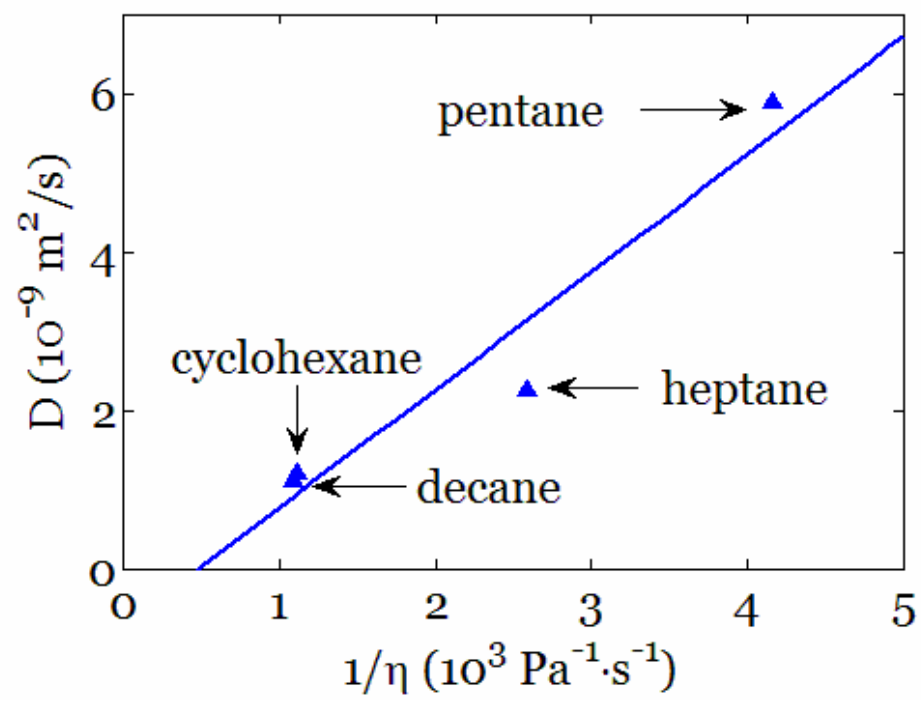

FIG. 8. Correlation between the viscosity of solvent in bulk liquid and diffusivity of the solvent in PDMS. 


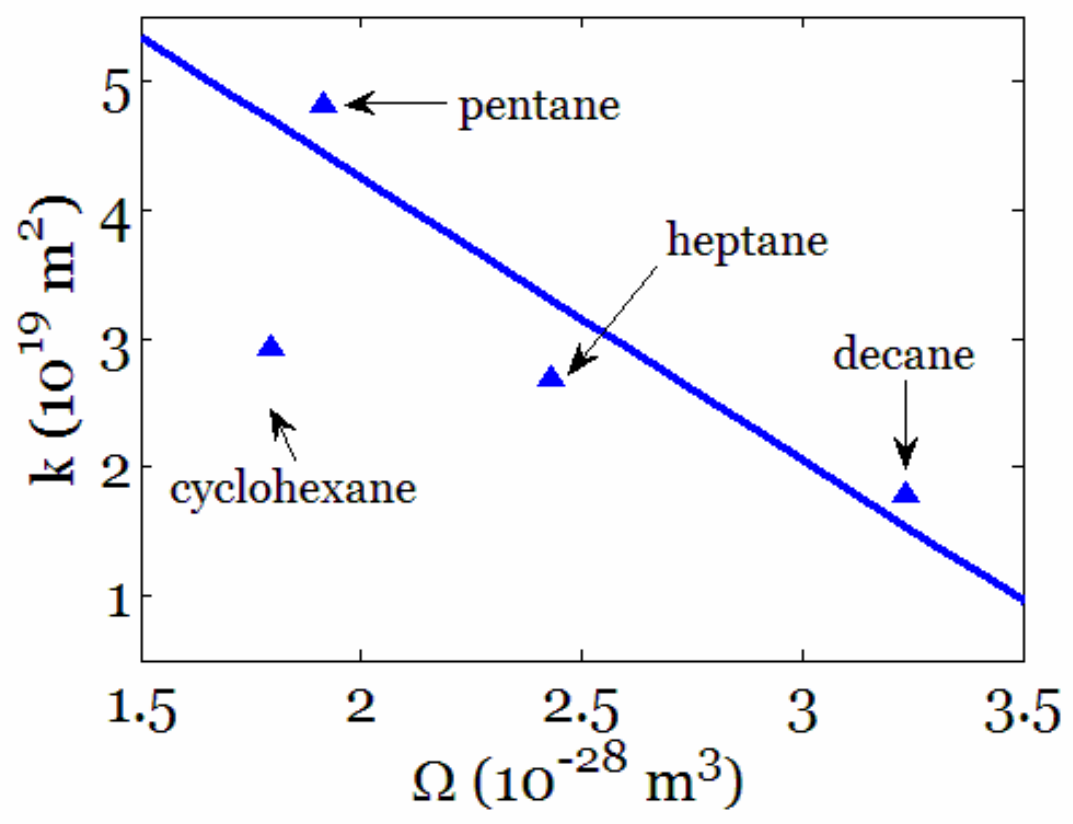

FIG. 9. Correlation between molar volume of solvent and diffusivity of the solvent in PDMS. 